\title{
Thermomechanical analysis of Natural Rubber behaviour stressed at room temperature.
}

\author{
R. Caborgan, J.-M. Muracciole ${ }^{\mathrm{a}}$, B. Wattrisse, A. Chrysochoos \\ Mechanics and Civil Engineering Laboratory, Montpellier University, Place E. Bataillon, 34095 \\ Montpellier Cedex 05, France
}

\section{1 abstract}

Owing to their high molecular mobility, stressed rubber chains can easily change their conformations and get orientated. This phenomena leads to so high reversible draw ratio that this behaviour is called rubber elasticity [1-3]. The analogy with ideal gases leads to an internal energy independent of elongation, the stress being attributed to a so-called configuration entropy.

However, this analysis cannot take thermal expansion into account and moreover prohibits predicting standard thermo-elastic effect noticed at small elongations and the thermoelastic inversion effects [4].

This paper aims at :

- $\quad$ observing and quantifying dissipative and coupling effects associated with deformation energy, generated when Natural Rubber is stretched.

- re-examine the thermomechanical behaviour model of rubberlike materials, under the generalised standard material concept.

From an experimental viewpoint, energy balance is created using infrared and quantitative imaging techniques. Digital Image Correlation (DIC) provides in-the-plane displacement fields and, after derivation, strain and strain-rate fields.

We have used those techniques to evidence the thermoelastic inversion effect as shown on Figure 1 where different weights have been fixed to warmed specimen and we monitored the sample deformation while it recovers room temperature.

But we have also used those techniques to perform energy balance : analysis of the mechanical equilibrium allows estimates of the stress pattern and computation of deformation energy rates under a plane stress hypothesis [5]. Infrared Thermography (IRT) gives the surface temperature of the sample. To estimate the distribution of heat sources, image processing with a local heat equation and a minimal set of approximation functions (image filtering) was used.

The time courses of deformation energy and heat associated with cyclic process are plotted in Figure 2. The time derivatives of both forms of energy are approximately similar. This is consistent with the fundamental hypothesis of the molecular theory of rubber elasticity.

To take into account those two thermomechanical couplings a strain tensor was introduced to characterize the configuration of the polymer chain network in terms of a new state variable in order

\footnotetext{
a e-mail : muracciole@Imgc.univ-montp2.fr
} 
to separate dissipative and coupling rubber mechanisms from standard thermo-elastic effects: comparison between experiments and simulation is shown on figure 1 where the circles are the experimental points and the line the model result. The results are shown

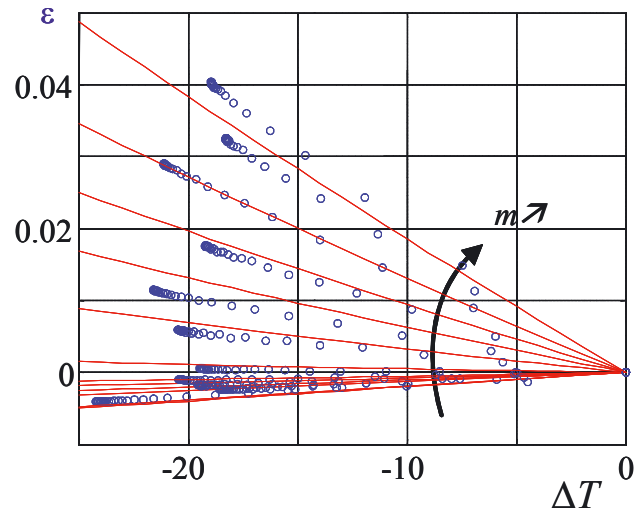

Fig. 1: thermoelastic inversion effect evidence: the strain evolution against temperature for different preloads (circles : experiment - line : modelling)

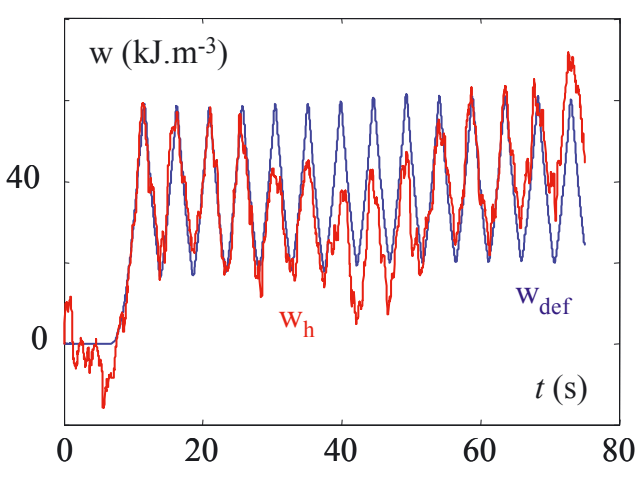

Fig. 2: Time course of heat and mechanical energy during cyclic loadings

\section{References}

1. L. R. G. Treloar. The physics of rubber elasticity. Oxford, Clarendon Press. (1975).

2. J. P. Joule, On some thermodynamic properties of solids, Philos. Trans. Roy. Soc. London: 91p.131, (1859).

3. R. L. Anthony, R. H. Caston and E. Guth . Equations of state for natural and synthetic rubberlike materials. J. Phys. Chem. 46, p.826. (1942).

4. P. Chadwick, C.F.M. Creasy, Modified entropic elasticity of rubberlike materials, J. Mech. Phys. Sol. 32, pp.337-397 (1984)

5. B. Wattrisse, A. Chrysochoos, J.-M. Muracciole, M. Nemoz-Gaillard, Analysis of strain localisation during tensile test by digital image correlation. $J$. of Exp. Mech., 41, n 1, pp. 29-38, (2000) 